\title{
The Impact of Carotid Angioplasty and Stenting on the Cerebrovascular Reactivity
}

\author{
Xinying Fan ${ }^{\text {a\# }}$ Huimin Zhu ${ }^{\text {a\# }}$ Xiangliang Chen ${ }^{\mathrm{a}}$ Gelin Xu $\mathrm{u}^{\mathrm{a}}$ Bernard Yan ${ }^{\mathrm{b}}$ Qin Yin $^{\mathrm{a}}$ \\ Yunyun Xiong ${ }^{\mathrm{a}}$ Xinfeng Liu ${ }^{\mathrm{a}}$ \\ aDepartment of Neurology, Jinling Hospital, Nanjing University School of Medicine, Nanjing, China; \\ ${ }^{b}$ Department of Neurology, Royal Melbourne Hospital, University of Melbourne, Melbourne, Australia
}

\section{Key Words}

Carotid artery $\cdot$ Stenosis $\cdot$ Stenting $\cdot$ Breath $\cdot$ holding index $•$ Follow-up

\begin{abstract}
Objective: To evaluate the effect of carotid angioplasty and stenting (CAS) on postoperative cerebrovascular reactivity (CVR) and its independent predictors. Methods: During July 2008 and September 2009, 37 patients undergone CAS were enrolled in this study. Breath holding tests using transcranial Doppler were performed for each patient before, 1 to 2 days, and 3 to 10 months after the procedure. CVR was evaluated by the breath holding index (BHI). Repeated measures analysis of variance was employed to detect the $\mathrm{BHI}$ changes after CAS. Multivariate linear regression was used to identify the independent predictors for $\mathrm{BHI}$. Results: A significant improvement of the ipsilateral $\mathrm{BHI}$ values was observed after stenting $(P<0.05)$. The follow-up $\mathrm{BHI}$ values were positively correlated with those after procedures $(\beta=1.030, P<0.001)$, while negatively correlated with tandem stenoses $(\beta=-0.016, P=0.002)$. Conclusions: CAS results in an immediate improvement and a gradual normalization of the ipsilateral CVR. Tandem stenoses are an independent predictor for CVR.
\end{abstract}

Copyright $\odot 2012$ S. Karger AG, Basel

\section{Introduction}

Carotid stenosis constitutes an important cause of ischemic cerebrovascular diseases. Revascularization of carotid stenosis benefits patients by preventing recurrent ischemic stroke. In recent years, driven by advancements in interventional technology and the accumulation of procedural expertise, carotid angioplasty and stenting (CAS) has become a valid approach for the treatment of carotid stenosis $[1,2]$. However, little is known on change of hemodynamics after stenting despite an emerging abundance of trails based on the efficacy and safety of CAS.

Transcranial Doppler (TCD) has been widely utilized to demonstrate flow signals of large intracranial arteries. When used in conjunction with the breath-holding test, it offers a reliable method for assessing cerebrovascular reactivity (CVR). As an important dynamic indicator of the cerebral circulation, CVR is representative of the cerebrovascular reserve capacity. It can be evaluated by calculating the breath-holding index (BHI), which is assessed by the mean blood flow velocity (MFV) changes of the middle cerebral artery (MCA) after hypercapnia induced by a breath hold. The aim of this study was to explore the effect of CAS on postoperative CVR, attempting to

"These authors contributed equally to this work. Second correspondence address: Bernard Yan, Department of Neurology, Royal Melbourne Hospital, University of Melbourne, Australia, E-Mail Bernard.yan@mh.org.au

\section{KARGER \\ Fax +41613061234 \\ E-Mail karger@karger.ch}

www.karger.com (c) 2012 S. Karger AG, Basel

$1015-9770 / 12 / 0341-0013 \$ 38.00 / 0$

Accessible online at:

www.karger.com/ced
Xinfeng Liu

Department of Neurology, Jinling Hospital

Nanjing University School of Medicine

305 Zhongshan East Road

Nanjing 210002 (China)

E-Mailxfliu2@yahoo.com.cn 
provide an objective index to evaluate the hemodynamic status after stenting.

\section{Subjects and Methods}

\section{Patients}

Patients undergoing CAS were enrolled between July 2008 and September 2009. Thirty-seven of 42 patients finished the followup, 26 were male and 11 were female. 3 patients were lost for follow up because of economic difficulty or emigration. There were 17 asymptomatic and 20 symptomatic patients. All the information was retrieved from Nanjing Stroke Registry System. The degree of carotid artery stenosis was measured according to the North American Symptomatic Carotid Endarterectomy Trial criteria. [3]

The symptomatic patients had ipsilateral transient ischemic attack (TIA) $(n=1,5 \%)$ and nondisabling stroke $(n=19,95 \%)$. A further magnetic resonance imaging showed that $55 \%$ of them had ipsilateral internal carotid artery (ICA) lacunar infarction $(n=11)$, $40 \%$ had non-lacunar infarction $(n=8)$; the one with TIA had no abnormal signal in the magnetic resonance image. Among the asymptomatic patients, there were 6 patients (35\%) with ipsilateral or bilateral ICA lacunar infarction, 11(65\%) with no lesion. Inclusion criteria were as follows: (1) $\geq 50 \%$ symptomatic stenosis at the ostial ICA proved by digital subtraction angiography (DSA), with or without contralateral lesions; or $\geq 70 \%$ asymptomatic ostial ICA stenosis on one or both sides. (2) Feasible bilateral temporal windows for TCD detection, and no evidence for occlusion of MCA.

Exclusion criteria: (1) Multiple stents were placed because of multi-stenosis. (2) Ipsilateral middle cerebral artery occlusion. (3) Clinical history of brain infarction. (4) Severe cardiac, pulmonary, renal diseases or uncooperative during the breath holding test. (5) Other contra-indications for stenting.

Meanwhile, we recruited 30 stroke-free patients as normal controls, mean age was $64.4 \pm 10.6$ years and 17 of them were male, Criteria of the control group included: (1) No stenosis at color Doppler ultrasonography, TCD, or the cervical CTA, MRA, or DSA; (2) MRI showed no acute or old infarction, and no severe ischemic changes of the brain; (3) Bilateral good temporal window for TCD.

\section{Methods}

Transcranial Doppler: Doppler examination was performed using a standard TCD system (Nicolet Companion III, Medilab, Estenfeld, Germany). The bilateral MCA was insonated with a 2.0$\mathrm{MHz}$ transducer probe through the temporal window, at a depth of $60 \mathrm{~mm}$ (56mm when bad signal); the Doppler frequency spectrum of the blood flow was then recorded. Breath holding test: the breath-hold training was performed beforehand. During the test, the patient in a supine position first took a quiet breathing for 4 to 5 minutes. When the MCA TCD frequency spectrum was detected, the probe remained in position, and the depth of the sampling volume remained stable, then patients were instructed to hold breath for about 30 seconds after a normal inspiration; the TCD frequency spectrum and the breath-holding time were recorded afterwards. Patients all took TCD breath holding tests at 1 day before, 1 to 2 days after CAS, and during follow-up. The $\mathrm{BHI}$ was calculated as the percentage increase in MCA mean blood velocity divided by seconds of breath-holding ( [Vbh - Vr/
Table 1. Characteristics of patients

\begin{tabular}{lc}
\hline Parameters & Frequency (\%) \\
\hline Male & $26(70 \%)$ \\
Age, mean (years) \pm SD & $62.9 \pm 12.4$ \\
Risk factors & $33(89 \%)$ \\
$\quad$ Hypertension & $16(43 \%)$ \\
Diabetes & $11(30 \%)$ \\
Cardiopathy & $15(41 \%)$ \\
Hyperlipidemia & $6(16 \%)$ \\
History of stroke & $16(43 \%)$ \\
Smoking & $20(54 \%)$ \\
Symptomatic patients &
\end{tabular}

$\mathrm{Vr}] \times 100 \times \mathrm{s}-1$ ), where $\mathrm{Vbh}$ was MCA mean blood velocity at the end of breath-holding, $\mathrm{Vr}$ the MCA mean blood velocity at rest, and $s-1$ per second of breath-holding.

DSA and CAS: A preoperative DSA examination was performed to determine the degree of artery stenosis, and the compensation of collateral circulation. CAS therapeutic strategy was then made according to the DSA results. DSA mainly involved angiography of the aortic arch, bilateral carotid artery, ICA and bilateral vertebral artery. The degree of carotid artery stenosis was measured according to the North American Symptomatic Carotid Endarterectomy Trial criteria [3].

\section{Statistical Analysis}

Data were processed by SPSS 13.0 software. Categorical data were compared using $\chi 2$ test, normal distributed quantitative data were presented as mean \pm SD. Repeated measures analysis of variance was used to measure the variances. Multivariate linear regression analysis was used to control the confounding factors of BHI (related symptoms, follow-up time, degree of stenosis, contralateral stenosis, number of collateral vessel, tandem stenoses, infarction area), so as to find the independent predictors for CVR. The restenosis factor is ignored because the restenosis rate was quite low (only 2 cases) in our study. A P $<0.05$ is considered to be statistically significant.

\section{Results}

\section{Characteristics of Patients}

From July 2008 to September 2009, 37 patients were enrolled. The demographic data of our study population is given in table 1 .

\section{DSA Results and General Information of Follow-Up}

DSA showed that all the patients had a stenosis of at least $50 \%$ on one or both sides of the ostial ICA. Of them, $21(57 \%)$ cases had contralateral carotid stenosis, 20 (54\%) cases had ipsilateral MCA or intracranial ICA stenosis (or tandem stenoses), 11 (24\%) cases had contralateral MCA stenosis, and 28 (76\%) had vertebrobasilar artery 
Fig.1. A typical CAS with a severe stenosis (75\%) in LICA angiography of posteroanterior projection. (a) The root of LICA stenosis of approximately 75\% (arrow). BHI was 0.52. (b) Angiograph after CAS. BHI was 0.85 .
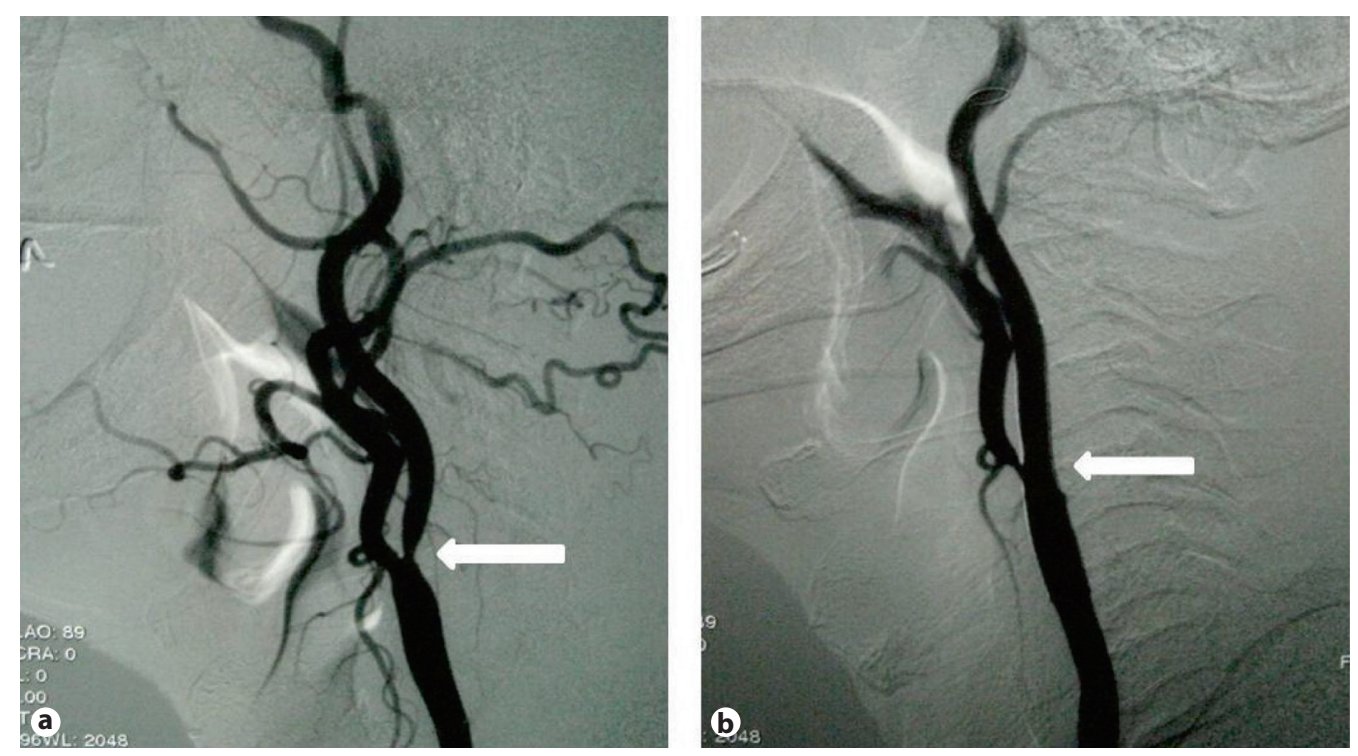

Table 2. Comparison of BHI values between ipsilateral and contralateral MCA before and after CAS and at the follow-up period (mean \pm SD)

\begin{tabular}{lllllrl}
\hline Group & No. of patients & Pre-CAS & Post-CAS & Follow-up & F value & P value \\
\hline Ipsilateral & 37 & $0.66 \pm 0.17$ & $1.01 \pm 0.10$ & $1.02 \pm 0.11$ & 58.900 & $<0.001$ \\
Contralateral & 37 & $0.93 \pm 0.28$ & $0.94 \pm 0.27$ & $0.95 \pm 0.25$ & 0.960 & $<0.001$ \\
Control & 30 & $1.09 \pm 0.11$ & $1.09 \pm 0.11$ & $1.09 \pm 0.11$ & - & - \\
\hline F value & & 29.860 & 0.911 & 1.432 & & \\
P value & & $<0.001$ & 0.415 & 0.248 & & \\
\hline
\end{tabular}

or posterior cerebral artery stenosis. No one underwent surgical or interventional treatment, and none of them worsened during follow-up. Mean follow-up time was $6.2 \pm 2.2$ months.

Eight of the 37 followed-up patients were re-hospitalized for dizziness and had a repeated DSA; another 2 cases had CAS on both sides sequentially ( 3 months in-between).

\section{BHI Comparison of Preoperative, Postoperative and \\ Follow-Up}

Before CAS, all the patients had a statistically significant reduction in BHI than the normal controls $(\mathrm{P}<0.001)$. The BHI of three groups (before, after CAS and during follow-up period) showed significant difference through repeated measures analysis of variance; the postoperative and follow-up BHI had a notable elevation than the preoperative group $(\mathrm{P}<0.05)$. Contralateral CVR showed a gradual improvement but not reach statistical significance $(0.93 \pm 0.28$ vs $0.94 \pm 0.27$ vs $0.95 \pm 0.25)$ (table 2, fig. 1).

\section{Predictors for BHI during Follow-Up}

The value of BHI during follow-up was differently affected by factors including the symptom, follow-up time, degree of stenosis, degree of contralateral stenosis, number of collateral vessel, tandem stenoses, infarction area, and so on. Multivariate linear regression analysis with stepwise method showed that the follow-up BHI values were positively correlated with CAS procedure $(\beta=1.030, P<0.001)$, whereas negatively correlated with tandem stenoses $(\beta=-0.016, \mathrm{P}=0.002)$.

\section{Discussion}

Atherosclerotic carotid artery stenosis is a main cause of TIA and cerebral infarction, which accounts for about 
$20 \%$ of ischemic events. It has the highest recurrence rate compared to other types of ischemic stroke. Effective interventional approach and medical therapy are essential by reducing the stroke incidence.

Carotid artery stenosis may result in embolic events in distal branches and hemodynamic disorder. Reduced hemodynamics leads to incomplete washout, and dilation in distal capillary vessel, then an impaired vasodilatory reserve, demonstrating a decreased or vanished CVR. CVR is an important cerebral hemodynamic indicator; it is predictive for prognosis and recurrence of ischemic cerebrovascular diseases. So it is meaningful to detect CVR in clinical work. The 2003 American guideline for perfusion imaging recommended the XeCT perfusion imaging with an acetazolamide challenge to screen chronic ischemic patients at high risk of recurrent cerebral infarction [4]. Powers found a strong relation of oxygen extraction fraction (OEF) with CVR, and $\mathrm{OEF}>0.52$ predicts reduced CVR, which means the risk of brain infarction is twice higher than normal [5]. At the same time, other studies demonstrated that severely impaired CVR was an independent predictor of recurrent ipsilateral TIA or infarction in MCA region [6]. TCD is a relative easy, quick and reliable way to evaluate CVR. An assessment of TCD from American academy of neurology pointed out that in patients with asymptomatic extracranial carotid artery occlusion, a BHI of $<0.69$ indicated impaired CVR and identified patients at risk for TIA and stroke [7]. Our study also showed that patients with moderate and severe carotid artery stenosis had a lower CVR compared to the contralateral and the control, indicating hemodynamic impairment and a potential benefit from CAS. Meanwhile, we observed a slight decrease in the contralateral CVR, probably owing to collateral flow compensation for the stenotic side.

CAS is performed mainly to prevent thromboembolic complications by stabilizing the ulcerated plaque; additionally, it normalizes cerebral hemodynamics by increasing the lumen diameter and providing a better cerebral irrigation. Markus et al. demonstrated that similar to carotid endarterectomy, carotid balloon dilation distinctly improved the ipsilateral CVR [8]. Sanchez-Arjona et al. showed that CVR was immediately improved after CAS, and maintained at 30 days after the procedure [9]. Brcic et al. found that the value of BHI was lower on the contralateral one day after stenting, but no significant difference was observed one month later, interpreted as one month of capillary bed adaptation to the dilated cerebral vessel. These showed that CAS can immediately improve the ipsilateral CVR after CAS, and reach normal value gradually. This was probably attributed to a recovery of normal diameter and a better cerebral irrigation, and therefore a normalized vasomotion function, eventually resulting in a notable increase of CVR within a short time. However, there would be no significant improvement of blood perfusion and vasomotion function over time, besides, the previous collateral vessel would regress, which all led to an unobvious change in CVR during follow-up. Our study demonstrated that CVR was markedly elevated after the procedure, but not evidently changed during follow-up, indicating that CAS can immediately improve CVR postoperatively, but can't make obviously further improvement over time.

Moreover, our study showed that the higher BHI after the procedure, the higher it would be during follow-up; while patients with tandem stenoses had lower BHI during follow-up. CVR can be affected by stenosis of either intracranial ICA or MCA. The conclusion drawn from multiple regression analysis may have bias due to a limited number of cases, selective bias (such as a relative mild symptom of patients undergoing CAS, a relative small area of infarction) as well as a small number and a mild degree of tandem stenoses and contralateral carotid artery stenosis.

In conclusion, CAS can improve the ipsilateral CVR after the procedure, and can also slightly increase the contralateral CVR; the higher BHI postoperatively, the higher it would be afterwards, while the more severe tandem stenoses, the lower BHI eventually. But due to a limited number of cases and a relative short time of follow-up, the long term impact of CAS still needs further study.

\section{Acknowledgements}

This study was supported by the Natural Science Foundation of China (NSFC \# 81171099 to WZ and NSFC \# 81070922 to GX).]

\section{Disclosure Statement}

The authors had no conflicts of interest to declare. 


\section{References}

1 Heck D: Thirty day results of 227 consecutive carotid stent procedures performed in carotid stenting clinical trials. J Neurointervent Surg 2009;1:154-158.

2 Li Shuibin, Yu Wanxiang, Cheng Gangwei: Stent-assisted angioplasty for carotid artery stenosis. Chin J Neuromed 2007;6:311-313.

3 Beneficial effect of carotid endarterectomy in symptomatic patients with high-grade carotid stenosis. North American Symptomatic Carotid Endarterectomy Trial Collaborators. N Engl J Med 1991;325:445-453.

-4 Latchaw RE, Yonas h, Hunter GJ, Yuh WT, Ueda T, Sorensen AG, Sunshine JL, Biller J, Wechsler L, Higashida R, Hademenos G: Guidelines and recommendations for perfusion imaging in cerebral ischemia: A scientific statement for healthcare professionals by the writing group on perfusion imaging, from the Council on Cardiovascular Radiology. Stroke 2003;34:1084-1104.
5 Powers WJ: Cerebral hemodynamics in ischemic cerebrovascular disease. Ann Neurol 1991;29:231-240.

6 Markus H, Cullinane M: Severely impaired cerebrovascular reactivity predicts stroke end TIA risk in patients with carotid artery stenosis and occlusion. Brain 2001;124:457-467.

7 Sloan MA, Alexandrov AV, Tegeler CH, Spencer MP, Caplan LR, Feldmann E, Wechsler LR, Newell DW, Gomez CR, Babikian VL, Lefkowitz D, Goldman RS, Armon C, Hsu CY, Goodin DS: Assessment: transcranial Doppler ultrasonography: report of the Therapeutics and Technology Assessment Subcommittee of the American Academy of Neurology. Neurology 2004;62: 1468-1481.

$>8$ Markus HS, Clifton A, Buckenham T, Taylor $\mathrm{R}$, Brown MM: Improvement in cerebral hemodynamics after carotid angioplasty. Stroke 1996;27:612-616.
9 Sanchez-Arjona MB, Sanz-Femandez G, Franco-Macias E, Gil-Peralta A: Cerebral hemodynamic changes after carotid angioplasty and stenting. AJNR Am J Neuroradiol 2007;28:640-644.

10 Brcic I, Homer S, Thaler D, Demarin V, Klein GE, Niederkorn K: Improved cerebral vasoreactivity following percutanenus transluminal angioplasty with stenting of high-grade internal carotid artery stenosis. Cerebrovasc Dis 2008;25:555-560. 